\title{
Family-based genome-wide association study for simulated data of Framingham Heart Study Hongyan $\mathrm{Xu}^{* 1}$, George Mathew ${ }^{2}$ and Varghese George ${ }^{1}$
}

\author{
Addresses: ${ }^{1}$ Department of Biostatistics, Medical College of Georgia, 1469 Laney Walker Boulevard, Augusta, Georgia 30912-4900, USA and \\ ${ }^{2}$ Department of Mathematics, Missouri State University, 901 South National Avenue, Springfield, Missouri 65897, USA \\ E-mail: Hongyan Xu* - hxu@mcg.edu; George Mathew - georgemathew@missouristate.edu; Varghese George - vgeorge@mcg.edu \\ ${ }^{*}$ Corresponding author
}

from Genetic Analysis Workshop 16

St Louis, MO, USA 17-20 September 2009

Published: 15 December 2009

BMC Proceedings 2009, 3(Suppl 7):SI24 doi: 10.1186/I753-656I-3-S7-SI24

This article is available from: http://www.biomedcentral.com/I753-656I/3/S7/SI 24

(c) $2009 \mathrm{Xu}$ et al; licensee BioMed Central Ltd.

This is an open access article distributed under the terms of the Creative Commons Attribution License (http://creativecommons.org/licenses/by/2.0), which permits unrestricted use, distribution, and reproduction in any medium, provided the original work is properly cited.

\begin{abstract}
Genome-wide association studies (GWAS) have quickly become the norm in dissecting the genetic basis of complex diseases. Family-based association approaches have the advantages of being robust to possible hidden population structure in samples. Most of these methods were developed with limited markers. Their applicability and performance for GWAS need to be examined. In this report, we evaluated the properties of the family-based association method implemented by ASSOC in the S.A.G.E package using the simulated data sets for the Framingham Heart Study, and found that ASSOC is a highly useful tool for GWAS.
\end{abstract}

\section{Background}

Genome-wide association studies (GWAS) are gaining popularity in genetic analysis of complex traits with the development of genotyping technology at the genome level. With genetic information at millions of singlenucleotide polymorphisms (SNPs) and other genetic markers, such studies offer great opportunities and also present challenges in developing appropriate statistical analysis methods. The data distributed by Genetic Analysis Workshop 16 provides a great opportunity to examine the strengths and limitations of current statistical methods for GWAS.

Many GWAS have been reported in the literature and many more are being performed. Most of them are population-based association studies and use designs such as case-control studies. Such designs have the advantages that samples are easy to ascertain and that results have relatively high power when analyses are carried out properly. However, it has been shown that population-based approaches, such as case-control studies, can produce spurious associations in the presence of population substructure, especially in large-scale studies at the genomic level [1,2]. An alternative approach is to use the family-based association methods, such as the transmission-disequilibrium test [3], the family-based association test (FBAT) [4], and a regression method [5] implemented by ASSOC in the S.A.G.E. package. These methods are robust to population substructure and other cryptic relatedness in the samples. However, these methods have been proposed in the era with only a few genetic markers and are intended mostly 
for candidate-gene studies. Their applicability and performance for GWAS has not been examined. The simulated data set of the Framingham Heart Study (FHS) provides both the family structure and the genotype information at genome-wide SNPs. The underlying simulation models are also provided. It is the purpose of this study to evaluate the performance of the familybased association method implemented by ASSOC in analyzing GWAS.

\section{Methods}

ASSOC implements a regression method that tests for association between a continuous trait and one or more covariates, including genetic markers from extended family data, and accounts for familial correlations [5]. The program estimates the parameters of a baseline model and those of alternate models that include specific sets of covariates. A likelihood-ratio test is then performed to evaluate the significance of the covariates.

The FHS simulated data set includes 6,476 subjects with both phenotype and genotype data in 942 pedigrees of up to three generations and 188 singletons. We excluded the singletons from our analysis because they do not have family information. The simulated data contain 200 replicates of the phenotypes. We used the phenotype data from the first replicate. Pedigree files were constructed from the supplied 'triplet_sim' file and merged with phenotype and genotype data. In addition to each of the SNPs, we included subject's age, smoking status, diet, and lipid-lowering drug usage as covariates. We kept all family members in the analysis because ASSOC could account for the familial correlations. We chose to test the association of HDL with all the makers from chromosome 19 in the $50 \mathrm{k}$ panel and $500 \mathrm{k}$ panel because there are two major genes for HDL on chromosome 19. In all, there are 1,639 markers in the $50 \mathrm{k}$ panel and 6,350 markers on $500 \mathrm{k}$ panel on chromosome 19. We assumed an additive model for all the markers. The program was run in a batch mode so that it would test for the association of the SNPs successively in one run. From the simulation model, there are five major genes for HDL. Two were on chromosome 19, and the other three were from chromosomes 8,9 , and 15 , respectively. The chromosome locations of the five major genes were given in Table 1. We tested the association of HDL with all the five major genes. There are a total of 1,000 polygenes for HDL, among which 15 are on chromosome 19.

\section{Results}

We performed association tests of all 1,639 markers on chromosome 19 with simulated HDL data. Out of 1,639 markers, the tests of 18 markers were significant at the 0.01 level. Because the HDL data were simulated using the markers in the $500 \mathrm{k}$ panel and not the markers in the $50 \mathrm{k}$ panel, and since the markers in the two panels are not close, we assume that the markers in the $50 \mathrm{k}$ panel did not contribute to the HDL phenotype. The significance result reflects false positives. Therefore, our results give an empirical type I error probability of 0.011 , which agrees well with the nominal level of 0.01 .

We further tested the association of the five SNPs representing the major genes with HDL (Table 1). They were all significant at 0.05 level regardless of the mode of inheritance and heritability. The true genetic models are additive for rs8103444, rs8035006, and rs8192719, and dominant for rs 10820738 and rsrs3200218. However, if more stringent significance level were used for genomewide studies, only two markers, rs10820738 and rs3200218, were found significant at $10^{-6}$ level. Table 1 also gives the estimated effect size for the five SNPs. SNP rs10820738 has the largest effect size, which is consistent with the simulation model in which it has the largest heritability.

We then tested all 6,350 SNP markers on chromosome 19 in the $500 \mathrm{k}$ panel for association with the simulated HDL data. Among the 15 markers that have polygenic effects on chromosome 19, association tests at six markers were significant at the 0.05 level (Table 2). The marker positions in base pairs are also given in Table 2 . The pairwise LD was relatively low, with a maximum $r^{2}$ of 0.003 as computed with Haploview [6]. A total of 395 tests were significant at 0.05 level for all the 6,350 markers tested on chromosome 19. Excluding the two major genes and six polygenes that are truly associated

Table I: Test results of the 5 SNPs from $500 \mathrm{k}$ panel for major gene effects of HDL

\begin{tabular}{lcccccccccc}
\hline Model & Location & Intercept & Diet & Sex & Rx $^{\mathbf{a}}$ & Age & Smoke $^{\mathbf{b}}$ & SNP & SE & $\boldsymbol{p}^{-V a l u e}$ \\
\hline rs10820738 & $9 \mathrm{q} 31.1$ & 68.694 & 0.028 & 13.565 & 1.486 & 0.019 & 0.714 & 4.524 & 0.518 & $3.20 \times 10^{-18}$ \\
rs3200218 & $8 \mathrm{p} 22$ & 68.680 & 0.027 & 13.552 & 1.420 & 0.020 & 0.664 & -1.671 & 0.319 & $1.63 \times 10^{-7}$ \\
rs8035006 & $15 \mathrm{q} 21$ & 68.684 & 0.023 & 13.527 & 1.474 & 0.020 & 0.647 & 0.831 & 0.284 & 0.003 \\
rs8192719 & $19 \mathrm{q} 13.2$ & 68.689 & 0.021 & 13.524 & 1.470 & 0.020 & 0.666 & -0.821 & 0.306 & 0.007 \\
rs8103444 & 19113.2 & 68.686 & 0.021 & 13.533 & 1.452 & 0.019 & 0.660 & 0.629 & 0.305 & 0.039 \\
\hline
\end{tabular}

${ }^{\mathrm{a}} \mathrm{Rx}$, lipid lowering drug use.

bSmoke, smoking status. 
Table 2: Test results among polygenes affecting HDL on chromosome 19

\begin{tabular}{|c|c|c|c|c|c|c|c|c|c|c|}
\hline Model & Position & Intercept & Diet & Sex & $\mathbf{R x}^{\mathbf{a}}$ & Age & Smoke ${ }^{b}$ & SNP & SE & $p$-Value \\
\hline rs 10420985 & $62,077,748$ & 68.693 & 0.017 & 13.519 & 1.508 & 0.020 & 0.656 & -1.693 & 0.457 & 0.000214 \\
\hline rs8182590 & $13,282,550$ & 68.691 & 0.029 & 13.542 & 1.478 & 0.019 & 0.686 & -0.973 & 0.263 & 0.000222 \\
\hline rs35I5088I & $2,572,193$ & 68.689 & 0.023 & 13.537 & 1.449 & 0.019 & 0.663 & -1.727 & 0.550 & 0.0017 \\
\hline rs8I07007 & $33,248,124$ & 68.691 & 0.022 & 13.548 & 1.454 & 0.020 & 0.651 & 1.683 & 0.537 & 0.001723 \\
\hline rs 10403702 & $44,840,424$ & 68.692 & 0.021 & 13.532 & 1.491 & 0.020 & 0.647 & 5.564 & 2.199 & 0.011418 \\
\hline rs725I886 & $46,880,615$ & 68.687 & 0.020 & 13.537 & $1.50 \mathrm{I}$ & 0.020 & 0.669 & -0.666 & 0.266 & 0.012645 \\
\hline rsII673050 & $35,535,929$ & 68.690 & 0.019 & 13.530 & 1.473 & 0.020 & 0.659 & -0.505 & 0.279 & 0.069854 \\
\hline rs2277987 & $8,458,273$ & 68.689 & 0.021 & 13.549 & 1.480 & 0.020 & 0.658 & 0.496 & 0.289 & 0.08626 \\
\hline rsl6989305 & $49,579,243$ & 68.689 & 0.023 & 13.540 & 1.482 & 0.020 & 0.666 & 4.904 & 3.111 & 0.115021 \\
\hline rs16966229 & $36,924,150$ & 68.687 & 0.021 & 13.530 & $1.46 \mathrm{I}$ & 0.020 & 0.660 & -2.165 & 1.381 & 0.117801 \\
\hline rs 17620029 & $24,146,152$ & 68.690 & 0.019 & 13.539 & 1.465 & 0.020 & 0.652 & -0.390 & 0.369 & 0.291036 \\
\hline rs378650l & $49,691,015$ & 68.691 & 0.021 & 13.536 & 1.473 & 0.020 & 0.656 & 0.217 & 0.314 & 0.490909 \\
\hline rsllo85876 & $13,947,5 \mid 3$ & 68.689 & 0.020 & 13.540 & 1.467 & 0.020 & 0.655 & -0.164 & 0.273 & 0.547852 \\
\hline rs I77|6486 & $34,455,727$ & 68.690 & 0.020 & 13.539 & 1.478 & 0.020 & 0.655 & -0.204 & 0.376 & 0.586957 \\
\hline rs599458 & $20,792,763$ & 68.689 & 0.021 & 13.538 & 1.473 & 0.020 & 0.657 & 0.082 & 0.454 & 0.856762 \\
\hline
\end{tabular}

${ }^{\mathrm{a}} \mathrm{Rx}$, lipid lowering drug use.

bSmoke, smoking status.

with the trait, this gives an estimated empirical type I error rate of 0.06 . Figure 1 gives the quantile-quantile plot of the $p$-values for all the null markers. The observed distribution fits well with the expected uniform distribution and there is no major inflation of type I error. Among the top 50 SNPs ranked by p-values, four SNPs are true findings with polygenic effects.

We also applied FBAT to test the association of HDL with all 6,350 SNPs on chromosome 19. A total of 325 tests are significant at 0.05 level. However, only two polygenes are true positives (rs10420985 and rs599458, with $p$-values 0.004408 and 0.028563 , respectively). None of the major genes was significant with

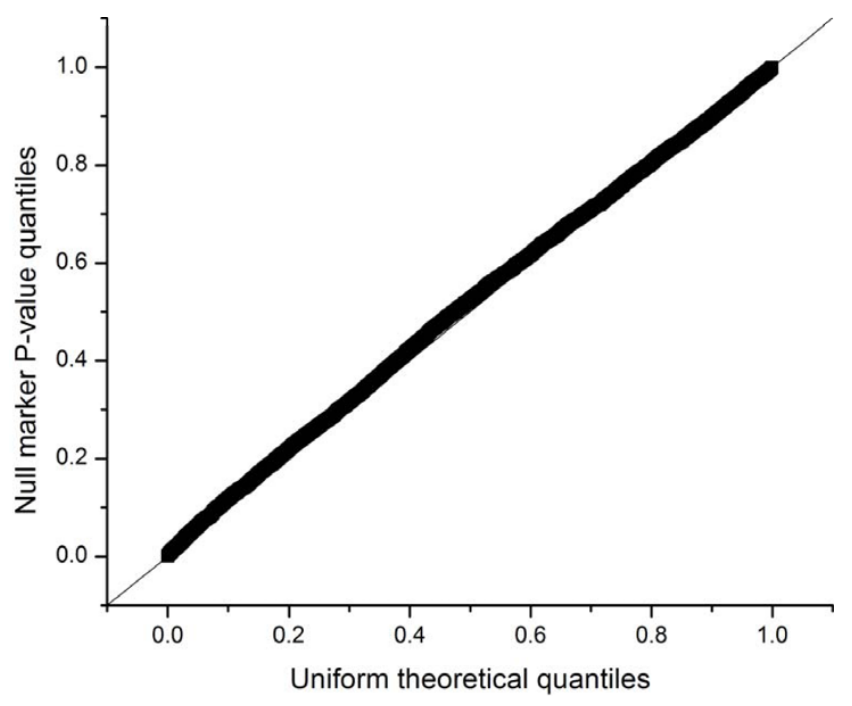

Figure I

Quantile-quantile plot of the p-values from null markers.
FBAT. Nonetheless, the type I error rate seems well controlled.

\section{Discussion}

Family-based association methods are appealing alternatives for the population-based case-control design because they are robust to population stratification in the samples. Several such methods have been proposed. However, they were all proposed before the current genomic era. As the norm of the field moves to GWAS, the performance and applicability of these methods need to be examined for GWAS. In this report, we examined the performance of a regression-based method [5], implemented in the program ASSOC in the software package S.A.G.E., using the simulated HDL data in the Framingham Heart Study. Based on the results of the tests with the markers on chromosome 19 in the $50 \mathrm{k}$ panel, we found that ASSOC gives the correct type I error rate. When applied to the markers on chromosome 19 in the $500 \mathrm{k}$ panel (tests performed at 0.05 level), the empirical type I error rate was 0.06, which is slightly inflated. The reason could be that there is linkage disequilibrium between the markers in the causal genes and markers close by, and when we estimated type I error rate, we only excluded the markers in the causal genes with either major or polygenic effects and not those markers in linkage disequilibrium. Therefore, as a general conclusion, ASSOC gives a more-or-less the correct type I error rate, and hence is a valid test for GWAS.

In our analysis, ASSOC detected all five major genes and six of the 15 polygenes for HDL on chromosome 19. In contrast, FBAT detected only two of the 15 polygenes and none of the major genes on chromosome 19. It should be noted that the data may be too limited to give 
a reliable estimation of the power. However, it is encouraging to see that ASSOC could detect one of the polygenes, rs10403702, whose minor allele frequency is only $0.35 \%$. Current association studies generally focus on common SNPs (e.g., SNPs with minor allele frequency $>5 \%$ ) based on the common disease, common variants hypothesis [7-10]. The other reason is that the statistical power may not be sufficient for rare SNPs when the sample size is limited. However, recent development in genotyping technology allows efficient genotyping in large samples and there is a call for shifting the paradigm of association studies to rare SNPs because it may be more effective to discover susceptibility genes for common diseases [11].

In conclusion, the method implemented in ASSOC provides a valid association test for family-based data and is reasonably powerful approach to be applied in GWAS. However, it should be noted that it is also a rather slow method. In our analysis, it took around 10 minutes to test one marker in our Windows-based workstation with $2.13 \mathrm{GHz}$ CPU. It will take substantial amount of time to perform the test for millions of markers in GWAS. Parallel computing would be the solution.

\section{Conclusion}

Using the simulated data for the Framingham Heart Study, we found the family-based regression method George et al. [5] implemented in ASSOC in the S.A.G.E. software is applicable to GWAS. It provides the correct type I error rate and reasonable power. However, this method is computationally time-consuming.

\section{List of abbreviations used}

FBAT: Family-based association test; FHS: Framingham Heart Study; GWAS: Genome-wide association studies; SNP: Single-nucleotide polymorphism.

\section{Competing interests}

The authors declare that they have no competing interests.

\section{Authors' contributions}

HX conceived of the study, performed the analysis, and drafted the manuscript. GM participated in the analysis and helped to draft the manuscript. VG participated in the design and coordination of the study. All authors read and approved the final manuscript.

\section{Acknowledgements}

The Genetic Analysis Workshops are supported by NIH grant ROI GM03 I575 from the National Institute of General Medical Sciences.
This article has been published as part of BMC Proceedings Volume 3 Supplement 7, 2009: Genetic Analysis Workshop 16. The full contents of the supplement are available online at http://www.biomedcentral.com/ $|753-656| / 3$ ? issue $=$ S7.

\section{References}

I. $\mathrm{Xu} \mathrm{H}$ and Shete S: Effects of population structure on genetic association studies. BMC Genet 2005, 6(SuppI I):SI09.

2. Marchini J, Cardon LR, Phillips MS and Donnelly P: The effects of human population structure on large genetic association studies. Nat Genet 2004, 36:5I2-5I7.

3. Spielman RS, McGinnis RE and Ewens WJ: Transmission test for linkage disequilibrium: the insulin gene region and insulindependent diabetes mellitus (IDDM). Am J Hum Genet 1993, 52:506-516.

4. Rabinowitz $D$ and Laird N: A unified approach to adjusting association tests for population admixture with arbitrary pedigree structure and arbitrary missing marker information. Hum Hered 2000, 50:2II-223.

5. George V, Tiwari HK, Zhu X and Elston RC: A test of transmission/disequilibrium for quantitative traits in pedigree data, by multiple regression. Am J Hum Genet 1999, 65:236-245.

6. Barrett JC, Fry B, Maller J and Daly MJ: Haploview: analysis and visualization of LD and haplotype maps. Bioinformatics 2005, 21:263-265.

7. Risch $\mathrm{N}$ and Merikangas $\mathrm{K}$ : The future of genetic studies of complex human diseases. Science 1996, 273:1516-1517.

8. Muller-Myhsok B and Abel L: Genetic analysis of complex diseases. Science 1997, 275:1328-1329.

9. Scott WK, Pericak-Vance MA and Haines JL: Genetic analysis of complex diseases. Science 1997, 275: I327.

10. Long $A D$, Grote $M N$ and Langley $C H$ : Genetic analysis of complex diseases. Science 1997, 275: 1328.

II. Gorlov IP, Gorlova OY, Sunyaev SR, Spitz MR and Amos Cl: Shifting paradigm of association studies: value of rare singlenucleotide polymorphisms. Am J Hum Genet 2008, 82: 100-II2.

\section{Publish with BioMed Central and every} scientist can read your work free of charge

"BioMed Central will be the most significant development for disseminating the results of biomedical research in our lifetime. "

Sir Paul Nurse, Cancer Research UK

Your research papers will be:

- available free of charge to the entire biomedical community

- peer reviewed and published immediately upon acceptance

- cited in PubMed and archived on PubMed Central

- yours - you keep the copyright 